\title{
Core Phase Transition of Radiation-Modified Polysilane Micelles as Revealed from Their Thermochromism
}

\author{
Hidenori TANAKA, ${ }^{1}$ Nobuhiro SATO, ${ }^{1, \dagger}$ Tomochika MATSUYAMA, ${ }^{1}$ \\ Rintaro INOUE, ${ }^{2}$ and Toshiji KANAYA ${ }^{2}$ \\ ${ }^{1}$ Research Reactor Institute, Kyoto University, Kumatori-cho, Sennan-gun, Osaka 590-0494, Japan \\ ${ }^{2}$ Institute for Chemical Research, Kyoto University, Uji 611-0011, Japan
}

(Received February 28, 2007; Accepted April 20, 2007; Published June 12, 2007)

\author{
KEY WORDS Polysilane / Micelle / Graft Copolymer / Thermochromism / $\gamma$-Rays / Radiation \\ Modification / \\ [doi:10.1295/polymj.PJ2006249]
}

The micelle formation of graft and block copolymers in selective solvents is an interesting topic on polymer self-assemblies. ${ }^{1,2}$ Although various micellar systems have been investigated, their detailed structure still remains to be clarified since micelles composed of at least two component chains are complex to examine discretely.

Recently, we reported solvatochromism of the micelles of poly(methyl acrylate)-grafted poly(methyl$n$-propylsilane) (PMPrS- $g$-PMA) and poly(acrylic acid)-grafted PMPrS (PMPrS-g-PAA) synthesized through $\gamma$-ray-induced graft polymerization. ${ }^{3}$ Polysilane is well-known for its chromic behavior ${ }^{4-8}$ caused by the main-chain conformational change as well as for its unique electronic and photonic properties. ${ }^{9}$ By taking advantage of the chromic changes of PMPrS, our former study disclosed the inner structure of the PMPrS- $g$-PAA micelles in water/tetrahydrofuran (THF) mixed solvents as follows. At water contents from 50 to $95 \%$, the swollen-core micelle formed, in which PMPrS chains in the micelle core are dissolved in THF and adopt the random conformation. At water contents above $95 \%$, the solidcore micelle formed, in which PMPrS core chains become solid and adopt the quasi-all-trans conformation.

In the present paper, we report core phase transition of PMPrS- $g$-PAA micelles in water/THF solvents as revealed from their thermochromism. The conformational transition of PMPrS core chains induced by a temperature change was closely examined by UV spectroscopy. In addition, light scattering measurements and a fluorescent method were also employed for clarifying the physical state of the micelles in the solution.

\section{EXPERIMENTAL}

\section{Synthesis}

PMPrS was synthesized via the Wurtz-type coupling reaction of distilled methyl- $n$-propyldichlorosilane (Shin-Etsu Chemical, Co.). GPC measurement showed that the number-average molecular weight of PMPrS used was 15000 against polystyrene standards.

PMPrS- $g$-PMA was synthesized through $\gamma$-ray-induced graft polymerization. PMPrS was dissolved together with methyl acrylate monomer in toluene and then irradiated with $\gamma$-rays after degassing. PMPrS$g$-PAA was prepared by the hydrolysis of PMA chains of PMPrS- $g$-PMA. The grafting yield (the number of grafted monomer units per silicon atom) was 0.33 for PMPrS- $g$-PAA determined from ${ }^{1} \mathrm{H}$ NMR signals. The details of preparation and characterization were reported in our previous paper. ${ }^{3}$

\section{Measurements}

PMPrS- $g$-PAA micelles were prepared in water/ THF mixed solvents. Water and THF are poor and good solvents for PMPrS, respectively. In a typical procedure of sample preparation, PMPrS- $g$-PAA was first dissolved in THF, and then a proper amount of water was added to give a desired solvent composition. Throughout this study, concentration of PMPrS$g$-PAA was fixed at $1.5 \times 10^{-2} \mathrm{~g} / \mathrm{L}$. A spin-coated film of PMPrS was prepared from a toluene solution on a quartz substrate.

Optical absorption and emission spectra were measured under controlled temperature. Temperature was measured by immersing a thermocouple in the solution or by attaching it directly to the film just before data acquisition. Static light scattering (SLS) and dynamic light scattering (DLS) measurements were car-

${ }^{\dagger}$ To whom correspondence should be addressed (Tel: +81-72-451-2499, Fax: +81-72-451-2633, E-mail: sato-n@ rri.kyoto-u.ac.jp). 
ried out with ALV-5000. The detection angle in the SLS measurements was $90^{\circ}$. Those in the DLS measurements were $30^{\circ}, 60^{\circ}, 90^{\circ}$ and $120^{\circ}$. The hydrodynamic size of the micelles was analyzed from DLS results by a single exponential fitting method.

\section{RESULTS AND DISCUSSION}

\section{UV absorption spectra of a PMPrS film}

Before investigating micelles, UV absorption spectra of a PMPrS spin-coated film were briefly examined for comparison. With increasing temperature from 20 to $50^{\circ} \mathrm{C}$, a thermochromic change was observed as seen in the literature. ${ }^{10}$ The absorption band peaking at $320 \mathrm{~nm}$ diminished and that peaking at $305 \mathrm{~nm}$ grew correlatively, which corresponds to the transition from the quasi-all-trans conformation to the random conformation of PMPrS chains. Here, we define transition temperature as the temperature at which the absorbance at $305 \mathrm{~nm}$ is equal to that at $325 \mathrm{~nm}$. The evaluated transition temperature of the PMPrS film, $46^{\circ} \mathrm{C}$, is in consistence with the literature value, ${ }^{10}$ and then this thermochromic behavior indicates melting of PMPrS chains. Thus, the conformational change in association with the phase transition was demonstrated to appear as the thermochromism.

\section{Temperature effect on solid-core micelles}

PMPrS- $g$-PAA forms solid-core micelles in a water /THF mixed solvent with a water content of $99 \%$ (in volume, likewise hereinafter) at room temperature as reported previously. ${ }^{3}$ The solid-core micelle consists of PMPrS core chains and PAA corona chains, where PMPrS chains are in the solid state and adopt the quasi-all-trans conformation at room temperature.

Figure 1 shows UV absorption spectra of PMPrS- $g$ PAA at a water content of $99 \%$ obtained with increasing temperature. The spectral behavior observed was

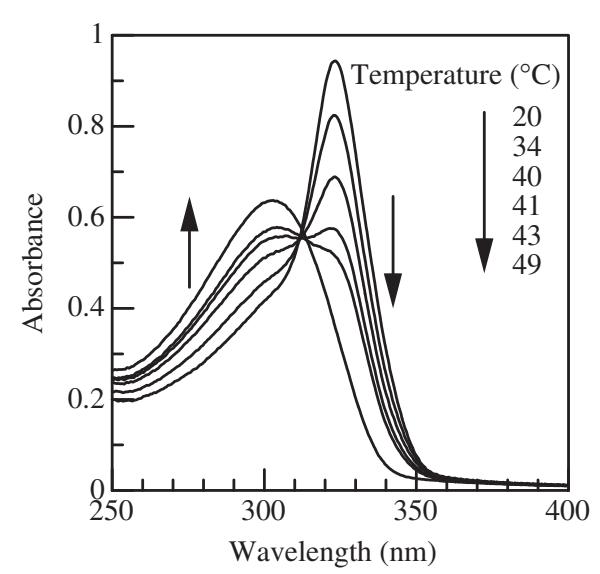

Figure 1. UV absorption spectra of PMPrS- $g$-PAA micelles at a water content of $99 \%$ measured with increasing temperature.

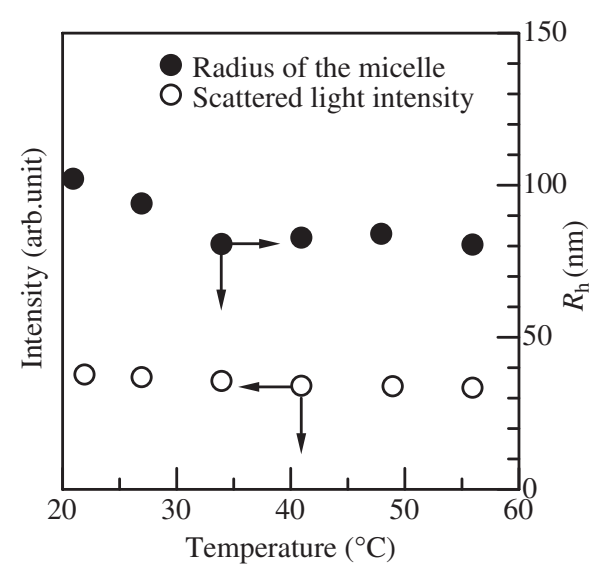

Figure 2. Temperature dependence of the static light-scattering intensity at $90^{\circ}(\bigcirc)$ and the hydrodynamic radius $\left(R_{\mathrm{h}}\right)$ of PMPrS- $g$-PAA micelles $(\bullet)$ at a water content of $99 \%$ evaluated from DLS.

parallel to that of the PMPrS spin-coated film and reversible with hysteresis upon a temperature change as described in the following section. The spectral transition indicates the conformational change of PMPrS chains from quasi-all-trans to random with rising temperature. Moreover, the transition temperature, $42^{\circ} \mathrm{C}$, is close to that of the PMPrS film. It is judged from this result that the solid core consisting of PMPrS chains changes into a molten core at this temperature.

The open circles in Figure 2 shows the SLS results of PMPrS- $g$-PAA micelles at a water content of $99 \%$ plotted against temperature. The scattering light intensity is almost constant in the shown temperature range, indicating that the aggregation number of the polymer chains in each micelle does not vary. ${ }^{11,12}$ This fact also indicates that the conformational transition demonstrated in Figure 1 is not caused by the dissolution of the micelles.

Next, the change in the micellar size was investigated by the DLS experiment with rising temperature. Also in Figure 2, the evaluated size is plotted by closed circles against temperature. Below the transition temperature, the micellar size becomes smaller with increasing temperature. This reduction of the size is interpreted in terms of the shrinkage of PAA corona chains because the hydrogen bonds between carboxyl groups of PAA and water molecules become weaker with rising temperature. ${ }^{11}$ Due to the breakage of hydrogen bonds, PAA corona chains are less solvated by water molecules and shrink with increasing temperature. Thus, the micellar size decreases as shown in the figure. Above the transition temperature, the size is almost constant. The reason for this is not fully clarified but we consider that it is due to the competition between the shrinkage of PAA corona chains and the increase of the core volume within the micelle. 
Since PMPrS core chains melt above the transition temperature as demonstrated above, the core volume should increase with the phase transition of PMPrS. This increase of the core volume compensates the corona shrinkage and the apparent size appears constant above the transition temperature.

When the temperature was risen from 20 to $60^{\circ} \mathrm{C}$ and then lowered to $20^{\circ} \mathrm{C}$ again, the micellar size was recovered. This thermal reversibility supports the idea that micellar structure is maintained after experiencing phase transition in the micelle core.

\section{Transition temperature and solvent composition}

PMPrS- $g$-PAA forms swollen-core micelles at water contents from 50 to $95 \%$ at room temperature. The swollen-core micelle contains THF within the core, which dissolves PMPrS chains. Fluorescence measurement using perylene proved that the swollen-core micelle becomes smaller with increasing water content. ${ }^{3}$

Thermochromic behavior at water contents of $95 \%$ and $89 \%$ is similar to that at a water content of $99 \%$, but the transition temperature varies as shown in Figure 3. The transition temperature lowers with decreasing water content. This result is explained by local concentration of PMPrS chains within the core. ${ }^{13}$ In the solid core, PMPrS chains contact with each other and therefore the local concentration of the PMPrS chains is high. The conformational transition of PMPrS chains requires large thermal energy because of the restriction of the chain motion. In the swollen core, on the contrary, the local concentration of PMPrS chains is lower than that of the solid core because THF partially solvates the core chains. The chain motion is less hindered and the conformational transition requires smaller energy. As a result, the

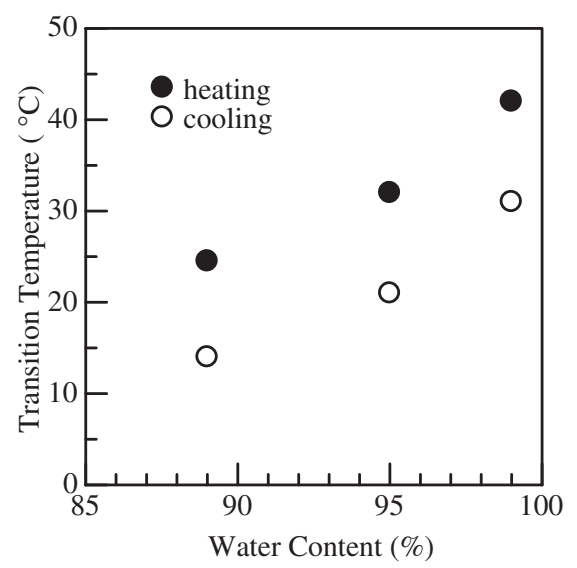

Figure 3. Relationship between the transition temperature and the solvent composition of PMPrS- $g$-PAA solutions. The data shown by and $\bigcirc$ are obtained in the heating and cooling process, respectively. transition temperature falls with decreasing water content as shown in Figure 3.

The transition temperature in the cooling process is always lower than that in the heating process as shown by open circles in Figure 3. Similar hysteresis was observed also for the PMPrS film: its transition temperature was $46^{\circ} \mathrm{C}$ on heating but $43.5^{\circ} \mathrm{C}$ on cooling. This is a supercooling phenomenon commonly found in polymer systems and that of polysilane is also reported in several studies by DSC measurements. ${ }^{8 c, 8 e}$

\section{Temperature effect on highly swollen micelles}

Lastly, we briefly discuss temperature effect on the swollen-core micelles that exhibited no phase transition. Below water contents of $69 \%$, the absorption spectra exhibited a peak around $305 \mathrm{~nm}$ and no spectral transition in the temperature range shown in Figure 3. This means no conformational transition of the PMPrS chains occur in this temperature range. Size information of the swollen-core micelles by the DLS measurement was also unavailable owing to the poor reproducibility of DLS data as also encountered in the previous study. Accordingly, we estimated the micelle structure by utilizing the excitation energy transfer method in the same manner as our former report. $^{3}$

The principle is as follow. When perylene is codissolved with the graft copolymer, hydrophobic perylene is incorporated in the micelle at low water contents. In this situation, energy transfer based on the Förster mechanism occurs from PMPrS chains to perylene molecules by selective excitation of PMPrS with $305 \mathrm{~nm}$ light, and thus fluorescence emission from perylene can be observed. If the core of micelles is shrinked, perylene emission should become stronger because the density of PMPrS chains increases and perylene can approach closer to PMPrS chains.

Figure 4 shows the emission intensity from pery-

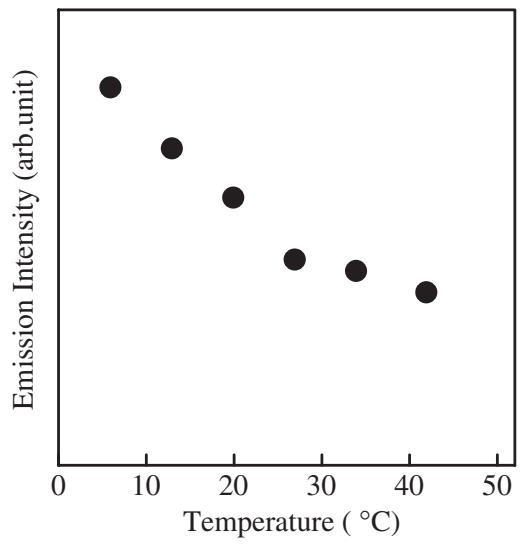

Figure 4. Emission intensity from perylene at $445 \mathrm{~nm}$ at a water content of $69 \%$. The concentration of perylene is $5.0 \times$ $10^{-3} \mathrm{~g} / \mathrm{L}$. 
lene at $445 \mathrm{~nm}$ observed for the micellar solution at a water content of $69 \%$. The emission intensity decreases with rising temperature. It is presumed that the Förster radius hardly changes with temperature because little change was observed for the spectral overlap between PMPrS emission and perylene absorption as well as for the emission intensity of PMPrS. Emission intensity from perylene alone in the solvent was also independent of temperature. Therefore, this decrease of the emission intensity is solely ascribable to the less efficient energy transfer due to extended distance between PMPrS chains and perylene molecules. This result indicates that the micelle core becomes more swollen because the solubility of PMPrS for THF increases with rising temperature. The graft copolymers more loosely aggregate in the micelles and get closer to the unimer state at higher temperature.

\section{CONCLUSION}

We disclosed the core phase transition of PMPrS- $g$ PAA micelles formed in water/THF mixed solvents. The core of the solid-core micelle transitions to the molten state with increasing temperature. Our pervious study revealed that there are two types of core states, that is, swollen and solid states depending on the solvent composition. The molten state is the third state that we found for the polysilane micelle, which is induced by a temperature rise. Through this study, we successfully demonstrated that the physical state of inner chains within micelles can be revealed by taking advantage of the optical property unique to polysilane. In the present system, temperature works as an external stimulus and the response appears as thermochromic behavior in UV absorption. It was proved thus that polysilane is expected to serve as a stimuli-responsive optical probe molecule.

\section{REFERENCES}

1. "Solvents and Self-Organization of Polymers," S. E. Webber, P. Munk, Z. Tuzar, and A. Arca, Ed., Kluwer,
Dordrecht, The Netherlands, 1996.

2. "Amphiphilic Block Copolymers: Self-Assembly and Application," P. Alexandridis and B. Lindman, Ed., Elsevier, Amsterdam, 2000.

3. H. Tanaka, N. Sato, and T. Matsuyama, Langmuir, 21, 7696 (2005).

4. a) K. Oka, N. Fujiue, S. Nakanishi, T. Takata, T. Dohmaru, C. H. Yuan, and R. West, Chem. Lett., 875 (1995).

b) K. Oka, N. Fujiue, T. Dohmaru, C. H. Yuan, and R. West, J. Am. Chem. Soc., 119, 4074 (1997).

5. a) C. H. Yuan and R. West, J. Chem. Soc. Chem. Commun., 1825 (1997).

b) S. Toyoda, M. Fujiki, C. H. Yuan, and R. West, Macromolecules, 33, 1503 (2000).

6. M. Fujino, T. Hisaki, and N. Matsumoto, Macromolecules, 28, 5017 (1995).

7. K. Song, H. Kuzumany, G. M. Wallraf, R. D. Miller, and J. F. Rabolt, Macromolecules, 12, 3870 (1990).

8. a) L. A. Harrah and J. M. Zeigler, J. Polym. Sci., Polym. Lett. Ed., 23, 209 (1985).

b) R. D. Miller, D. Hofer, J. Robolt, and G. N. Fickes, J. Am. Chem. Soc., 107, 2172 (1985).

c) J. F. Rabolt, D. Hofer, R. D. Miller, and G. N. Fickes, Macromolecules, 19, 611 (1986).

d) K. Sakamoto, M. Yoshida, and H. Sakurai, Macromolecules, 23, 4494 (1990).

e) C. H. Yuan and R. West, Macromolecules, 31, 1087 (1998).

f) T. Sanji, K. Sakamoto, H. Sakurai, and K. Ono, Macromolecules, 32, 3788 (1999).

9. For reviews, see: a) R. J. West, J. Organomet. Chem., 300, 327 (1986).

b) R. D. Miller and J. Michl, Chem. Rev., 89, 1359 (1989).

10. W. Chunwachirasiri, I. Kanaglekar, M. J. Winokur, J. C. Koe, and R. West, Macromolecules, 34, 6719 (2001).

11. S. Pispas and N. Hadjichristidis, Macromolecules, 36, 8732 (2003).

12. The association number of the micelle was not determined from light scattering measurement, but if the micelle is approximated by the same-size solid sphere with the density of PMPrS- $g$-PAA, $1.0 \mathrm{~g} / \mathrm{cm}^{3}$, then the association number of the solid-core micelle is estimated as $1.4 \times 10^{5}$. A similar treatment is also found in the literature (L. Zhang and A. Eisenberg, J. Am. Chem. Soc., 118, 3168 (1996).).

13. K. Furukawa, K. Ebata, D. Ichikawa, and N. Matsumoto, Macromolecules, 36, 7681 (2003). 